\title{
The problem of kinematic mass corrections for unpolarized semi-inclusive deep inelastic scattering
}

\author{
Ekaterina Christova* and Elliot Leaden $\dagger^{\dagger}$ \\ Institute for Nuclear Research and Nuclear Energy \\ Sofia, Bulgaria and \\ Blackett laboratory \\ Imperial College London \\ London SWr 2AZ, UK
}

(Dated: November 11, 2018)

\begin{abstract}
Miraculously, target mass corrections for inclusive deep inelastic scattering can be calculated exactly. On the contrary, there does not exist a consistent derivation of kinematic hadron mass corrections for semi-inclusive deep inelastic scattering (SIDIS). Recently this has become of topical interest, since there is a significant difference between the measured HERMES and COMPASS pion and kaon multiplicities, which cannot be explained as a consequence of evolution in $Q^{2}$, and it has been suggested that the difference can be understood if kinematic hadron mass corrections are taken into account. We explain why this argument is incorrect.
\end{abstract}

PACS numbers: 11.80.Cr, 12.38.-t, 13.60.Hb, 14.20.Dh

* echristo@inrne.bas.bg

$\dagger$ e.leader@imperial.ac.uk 


\section{INTRODUCTION}

Historically, the derivations of target-mass corrections (TMC) for inclusive deep inelastic scattering (DIS) were all based on the operator product expansion (OPE). The results for unpolarized DIS were first derived by Nachtmann [1] employing a very elegant mathematical approach in which the power series expansion used in the OPE was replaced by an expansion into a series of hyperspherical functions (representation functions of the homogeneous Lorentz group). Later, also within the context of the OPE, Georgi and Politzer [2] re-derived Nachtmann's results using what they called an alternative analysis "for simple-minded souls like ourselves" i.e. based on a straightforward power series expansion but, in fact, requiring a very clever handling of the combinatoric aspects of the problem.

The derivation of target-mass corrections for polarized DIS turned out to be much more difficult. Several papers [3, 4] succeeded in expressing the reduced matrix elements $a_{n}, d_{n}$ of the relevant operators in terms of combinations of moments of the structure functions, but did not manage to derive closed expressions for the structure functions $g_{1,2}$ themselves. The latter was finally achieved in 1997 by Piccione and Ridolfi [5] and later generalized to weak interaction, charged current reactions, by Blümlein and Tkabladze [6].

Semi-inclusive deep inelastic scattering reactions, where a final-state hadron is monitored, are of great interest, since they allow the extraction of information about individual antiquark distributions, and there is a major experimental effort underway to study them. However much of the most accurate data is, and will be for the forseeable future, in the kinematic region of relatively low $Q^{2}$, and it is thus of importance to know the kinematic hadron mass corrections (HMC) resulting from taking into account the target mass and produced hadron mass in these reactions.

The problem faced in deriving HMC for semi-inclusive deep inelastic scattering (SIDIS) is that the OPE does not apply. For this reason, D'Alesio, Leader and Murgia searched for a method which does not rely on use of the OPE and showed how the exact TMC for DIS, both unpolarized and polarized, could be derived in a totally different approach [7]. They made the crucial observation that TMC, by definition, are kinematic corrections, and therefore cannot depend on the numerical value of the strong interaction coupling $g$. Thus they can be calculated exactly with $g=0$ i.e. using the "handbag" diagram as shown in Fig. 1. 


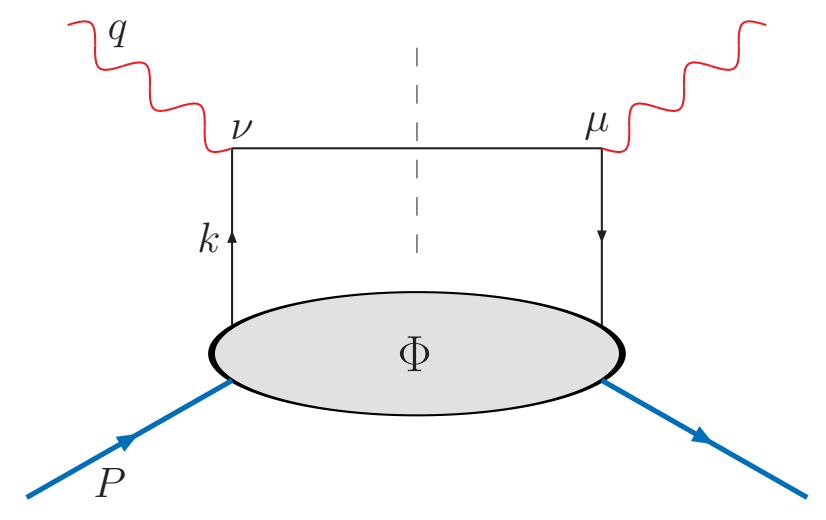

FIG. 1. The DIS "handbag" diagram involving the $q q$-correlator.

Christova and Leader (CL) thus attempted to apply this approach to calculate the exact HMC, to order $1 / Q^{2}$, for unpolarized SIDIS [8]. Unfortunately they found that there are serious problems and that the results break gauge invariance at the level of $(\text { mass })^{2} / Q^{2} 1$ Moreover, as will be explained, it seems clear that this problem is not linked to the use of $g=0$ and is of a more fundamental nature.

Recently Guerrero, Ethier, Accardi, Casper and Melnitchouk (GEACM) 9] presented a derivation of HMC for SIDIS and suggested that taking into account the HMC reduces, to a large extent, the difference between the HERMES and COMPASS pion and kaon multiplicities [10]. Unfortunately, as we shall show, the GEACM derivation is inconsistent.

\section{NOTATION AND CONVENTIONS}

We shall largely follow the conventions of the classic paper (LM) of Levelt and Mulders [11]. We consider the SIDIS reaction

$$
e\left(k_{e}\right)+N(P) \rightarrow e\left(k_{e}^{\prime}\right)+h\left(P_{h}\right)+X
$$

where $N$ is the nucleon of mass $M, h$ is the detected hadron of mass $M_{h}$ and $X$ is the remainder of the final state. We use the standard DIS variables with $E$ and $E^{\prime}$ the initial and final lepton energies in the target rest frame.

$$
Q^{2}=-q^{2} \quad \nu=\frac{P \cdot q}{M}=E-E^{\prime} \quad x_{B}=\frac{Q^{2}}{2 M \nu} \quad y=\frac{P \cdot q}{P \cdot k_{e}}=\frac{\nu}{E}
$$

\footnotetext{
${ }^{1}$ For this reason we did not attempt to publish our work of 2011.
} 


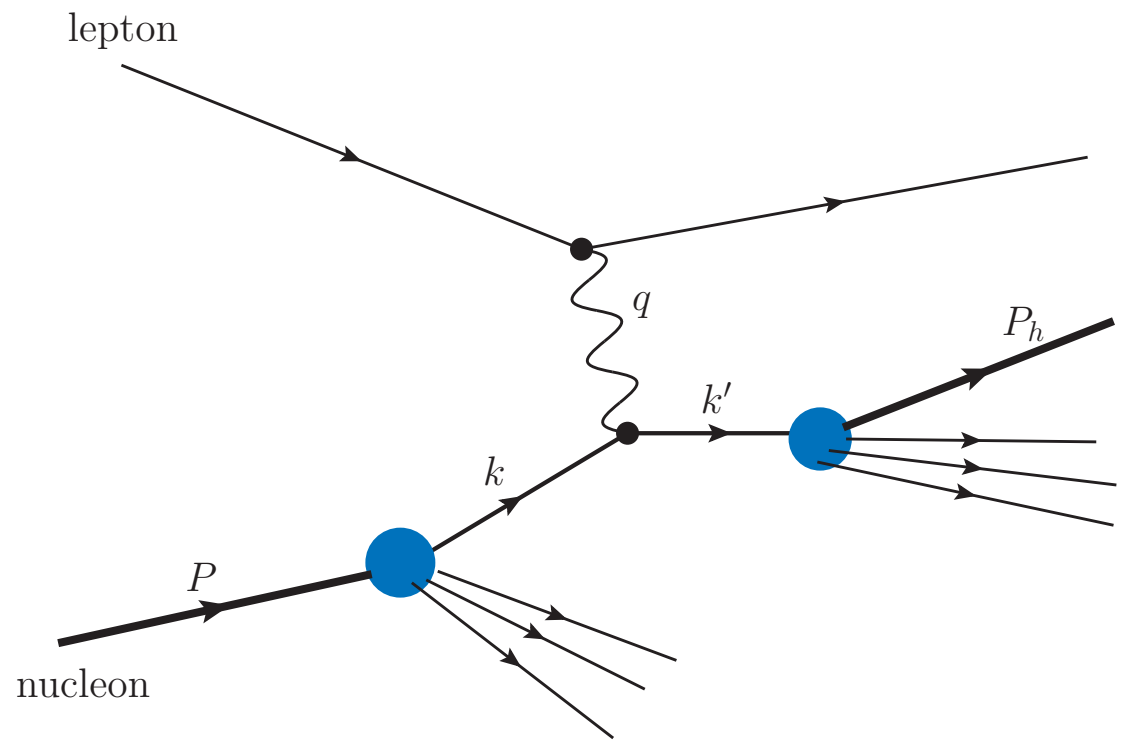

FIG. 2. The conventional partonic diagram for semi-inclusive lepton-nucleon reactions.

and the usual fragmentation variable $z_{h}$ defined as

$$
z_{h}=\frac{P \cdot P_{h}}{P \cdot q}=\frac{E_{h}}{\nu}
$$

where $E_{h}$ is the energy of the produced hadron in the target rest fame. The hadronic tensor for inclusive DIS is denoted by $W^{\mu \nu}$, and for semi-inclusive DIS by $\mathcal{W}_{h}^{\mu \nu}$. The particle label $h$ will occasionally be left out for typographical clarity.

The unpolarized SIDIS cross-section is given by

$$
\frac{2 E_{h} d \sigma}{d^{3} P_{h} d x_{B} d y}=\frac{\pi \alpha^{2} y}{Q^{4}} L_{\mu \nu} \mathcal{W}_{h}^{\mu \nu}
$$

and the spin-averaged leptonic tensor is

$$
L^{\mu \nu}=2 k_{e}^{\mu} k_{e}^{\prime \nu}+2 k_{e}^{\nu} k_{e}^{\prime \mu}-Q^{2} g^{\mu \nu} .
$$

\section{EXPRESSION FOR SIDIS HADRONIC TENSOR $\mathcal{W}_{h}^{\mu \nu}$ IN TERMS OF QUARK CORRELATORS}

From Fig. 2, for a quark of charge $e_{q}$, for the unpolarized case we have:

$$
\mathcal{W}_{u n}^{\mu \nu}\left(P, P^{h}, q\right)=e_{q}^{2} \int d^{4} k d^{4} k^{\prime} \delta^{4}\left(k+q-k^{\prime}\right) \operatorname{Tr}\left[\gamma^{\mu} \Phi_{q} \gamma^{\nu} \Delta_{q}^{h}\right]
$$


where $\Phi_{i j}^{q}(P, k)$ is the spin-independent quark production correlator, with $k^{\mu}$ the 4-momentum of the active quark, and $i, j$ Dirac indices, and $\Delta_{q}^{h}\left(P_{h}, k^{\prime}\right)$ is the spin-independent quark fragmentation correlator, with $k^{\prime}=k+q$ the momentum of the fragmenting quark. It is important, as will become clear presently, to keep separate the virtualities of the the active quark and the fragmenting quark. We shall label these virtualities $m_{q}^{2}$ and $m_{q}^{\prime 2}$ i.e. we take

$$
k^{2} \equiv\left(m_{q}\right)^{2} \quad \text { and } \quad k^{\prime 2} \equiv\left(m_{q}^{\prime}\right)^{2}
$$

In the usual treatment, where all hadron masses are ignored, one takes $m_{q}=m_{q}^{\prime}=0$, and finds, that the leading twist expression for the SIDIS differential cross-section, takes the form, for each flavour,

$$
\frac{d \sigma}{d x_{B} d y d z_{h}} \propto q\left(x_{B}\right) D_{q}^{h}\left(z_{h}\right)
$$

where $q\left(x_{B}\right)$ is the usual quark-parton density $(\mathrm{PDF})$ and $D_{q}^{h}\left(z_{h}\right)$ the standard fragmentation function $(\mathrm{FF})$.

This result follows upon utilizing the leading twist expressions, which we shall refer to as "order 1" i.e. $O(1)$, namely

$$
\begin{gathered}
\Phi_{q}\left(x_{B}\right) \equiv \int d k^{-} d^{2} \boldsymbol{k}_{\perp} \Phi_{q}(P, k)=\frac{1}{2} q\left(x_{B}\right) \not h \\
\Delta_{q}^{h}\left(z_{h}\right) \equiv \int d k^{\prime} d^{2} \boldsymbol{k}_{\perp}^{\prime} \Delta_{q}^{h}\left(P_{h}, k^{\prime}\right)=\frac{1}{2} D_{q}^{h}\left(z_{h}\right) \not h,
\end{gathered}
$$

where the GEACM null vectors are defined as 2

$$
\bar{n}^{\mu}=\frac{1}{\sqrt{2}}(1,0,0,1) \quad \text { and } \quad n^{\mu}=\frac{1}{\sqrt{2}}(1,0,0,-1),
$$

and neglecting terms of $O(\mathcal{M} / Q)$ and $O\left(\mathcal{M}^{2} / Q^{2}\right)$, where $\mathcal{M}^{2}$ generically stands for $M^{2}, M_{h}^{2}$ or $M M_{h}$, when evaluating $\mathcal{W}_{u n}^{\mu \nu}\left(P, P^{h}, q\right)$ in Eq. ([6)

\section{THE APPROACH OF GEACM AND ITS PROBLEMS}

In their treatment of the HMC, GEACM utilize Eqs. (9, 10) in Eq. (6), but assume collinear production i.e. put $\boldsymbol{P}_{\perp}^{h}=0$, arguing that the transverse momentum should be

\footnotetext{
2 These null vectors are almost universally labeled $n_{+}$and $n_{-}$in the literature.
} 
generated by interactions, and then treat the kinematics in Eq. (6) more carefully, keeping all terms of $O\left(\mathcal{M}^{2} / Q^{2}\right)$. Their key result is that Eq. (8) is then replaced by

$$
\frac{d \sigma}{d x_{B} d y d z_{h}} \propto q\left(\xi_{h}\right) D_{q}^{h}\left(\zeta_{h}\right)
$$

where

$$
\xi_{h}=\xi\left(1+\frac{\left(m_{q}^{\prime}\right)^{2}}{Q^{2}}\right)
$$

and

$$
\zeta_{h}=\frac{z_{h} \xi}{2 x_{B}}\left(1+\sqrt{1-\frac{4 x_{B}^{2} M^{2} M_{h}^{2}}{z_{h}^{2} Q^{4}}}\right) .
$$

Here $\xi$ is the usual Nachtmann variable:

$$
\xi=\frac{2 x_{B}}{1+\sqrt{1+4 x_{B}^{2} M^{2} / Q^{2}}} .
$$

It is clear that the GEACM result differs from the conventional massless result by terms of order $O\left(\mathcal{M}^{2} / Q^{2}\right)$. Thus to be consistent and believable the GEACM evaluation of $\mathcal{W}_{u n}^{\mu \nu}\left(P, P^{h}, q\right)$ in Eq. (6) must be correct to $O\left(\mathcal{M}^{2} / Q^{2}\right)$. Now $\mathcal{W}_{u n}^{\mu \nu}\left(P, P^{h}, q\right)$ involves a product of $\Phi_{q}$ and $\Delta_{q}^{h}$ so that to achieve the desired accuracy each of $\Phi_{q}$ and $\Delta_{q}^{h}$ must be given correct to $O\left(\mathcal{M}^{2} / Q^{2}\right)$. But this is not done! Eqs. (9, 10) are only correct to $\mathrm{O}(1)$. Hence the GEACM result is not consistent.

We shall now indicate the, what to us seem like insurmountable difficulties, that arise if we try to remedy this problem in a straightforward way.

Correct to $O\left(\mathcal{M}^{2} / Q^{2}\right)$ the quark production correlator involves 3 scalar functions and has the form:

$$
\Phi_{q}(x)=\frac{M}{2 P_{+}} e(x)+\frac{q(x)}{2} \not h+\frac{M^{2}}{2\left(P_{+}\right)^{2}} b(x) \not h
$$

where, in what GEACM call the Breit Frame, $P_{+}=O(Q)$. It might be thought that the extra functions appearing in Eq. (16) are a consequence of interactions and therefore can be ignored in a purely kinematic analysis , but according to Mulders and Tangerman [12] this is incorrect. They show that e.g.

$$
e(x)=e_{k i n}(x)+e_{\text {int }}(x)
$$

where

$$
e_{k i n}(x)=\frac{m_{q}}{x M} q(x) .
$$


A completely analogous development holds for the fragmentation correlator $\Delta_{q}^{h}$, which then also contains 3 terms, parts of which are definitely not due to interaction.

The most serious consequence of using $\Phi_{q}(x)$ and $\Delta_{q}^{h}$, correct to $O\left(\mathcal{M}^{2} / Q^{2}\right)$, is the breakdown of gauge invariance for $\mathcal{W}_{u n}^{\mu \nu}\left(P, P^{h}, q\right)$, which we will now explain.

\section{THE BREAKDOWN OF GAUGE INVARIANCE: A SIMPLE DEMONSTRA- TION}

We are only interested in kinematical corrections. A simple trick to isolate these is thus to switch off the strong interaction i.e. to take $\alpha_{s}=0$. Then, according to [7], the expressions for the corrected correlators become

$$
\begin{gathered}
\Phi_{q}(x) \propto q(x)\left[m_{q}+\not k\right] \\
\Delta_{q}^{h}(z) \propto D_{q}^{h}(z)\left[m_{q}^{\prime}+\not k^{\prime}\right]
\end{gathered}
$$

which lead to

$$
\mathcal{W}_{u n}^{\mu \nu} \propto\left(m_{q} m_{q}^{\prime}-k \cdot k^{\prime}\right) g^{\mu \nu}+\left(k^{\mu} k^{\prime \nu}+k^{\nu} k^{\prime \mu}\right)
$$

Gauge invariance requires that

$$
q_{\mu} W_{u n}^{\mu \nu}=0
$$

Using Eq. (21) we find

$$
q_{\mu} W_{u n}^{\mu \nu} \propto\left(m_{q}^{\prime}-m_{q}\right)\left[m_{q} q^{\nu}+\left(m_{q}^{\prime}+m_{q}\right) k^{\nu}\right]
$$

In other words, gauge invariance demands that $m_{q}=m_{q}^{\prime}$. Is this possible?

In the standard treatment, ignoring hadron masses, one conventionally takes $m_{q}=m_{q}^{\prime}=0$ and gauge invariance is fine. When hadron masses are included there are compelling reasons to still choose $m_{q}=0$, as GEACM do, but it is certainly incorrect to take $m_{q}^{\prime}=0$. Indeed, kinematical considerations imply a lower bound for $m_{q}^{\prime 2}$. For the collinear case CL also have it:

$$
m_{q}^{\prime 2} \geq M_{h}^{2} / z_{h}
$$

which is compatible with the CEACM lower bound $m_{q}^{\prime 2} \geq M_{h}^{2} / \zeta_{h}$. 3 This immediately implies that we cannot take $m_{q}=m_{q}^{\prime}$ and we are forced to conclude that when terms of

${ }^{3}$ The GEACM result assumes that the target remnant jet has (mass $)^{2} \equiv(P-k)^{2} \geq M^{2}$, an assumption which we don't think can be justified, given that the remnant jet has baryon number $2 / 3$ and is coloured, and which disagrees with the condition $(P-k)^{2} \geq 0$ used by Ellis, Furmanski and Petronzio [13]. 

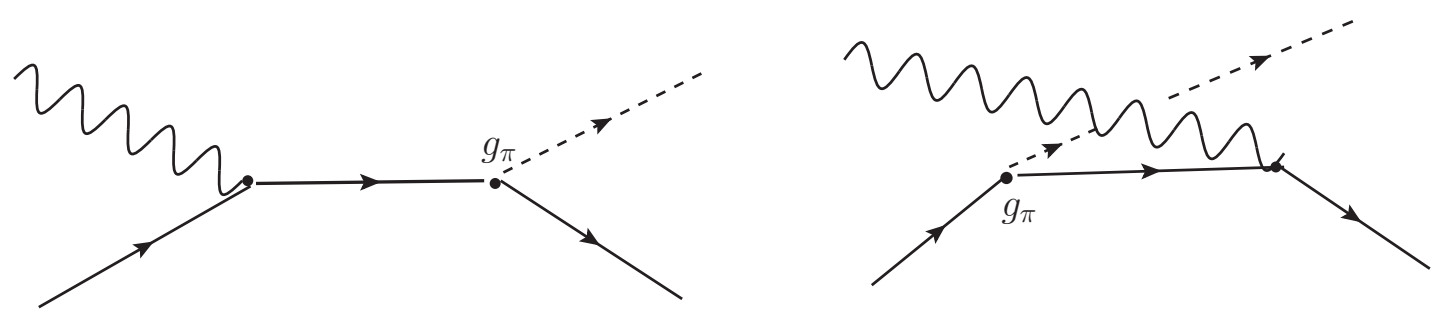

FIG. 3. Feynman diagrams for pion photoproduction on a quark. Only the sum of both diagrams is gauge invariant. The analogue of the crossed diagram is missing in Fig. 2.

$O\left(\mathcal{M}^{2} / Q^{2}\right)$ are included consistently in the GEACM appraoach, the result is not gauge invariant.

(In addition to this problem, there is another worrying matter. In their paper GEACM choose the particular value $M_{h}^{2} / \zeta_{h}$ for $m_{q}^{\prime 2}$. But any value larger than this would be acceptable. Hence there is effectively an arbitrary parameter in the GEACM treatment.)

Consider now the implications of the lower bound in Eq. (24). As stressed by Mulders and Tangerman [12] the validity of the parton model in QCD depends on the assumption that all the quark correlators cut off rapidly with increasing quark virtuality, implying that the fragmentation correlator should cut off rapidly with increasing $k^{\prime 2} \equiv m_{q}^{\prime 2}$. But use of Eq. (24) to describe experimental multiplicity values which are not small for small values of $z_{h}$ would imply that the correlator is large for virtualities much greater than a (hadrom mass) ${ }^{2}$.

\section{POSSIBLE RESOLUTIONS OF THE PROBLEM??}

The above discussion suggests that there is no way to accommodate non-zero hadron masses in the conventional treatment of SIDIS reactions. We list here, with some lack of conviction, a couple of unconventional ways to overcome the difficulties.

1. Since the virtuality of the fragmenting quark is considerably larger than the square of a typical hadron mass, it is neither a typical partonic quark nor a constituent quark. It is therefore some kind of effective quark and as such one might introduce an effective electromagnetic coupling e.g.

$$
\gamma^{\mu} \rightarrow \gamma^{\mu}-\frac{\not q}{q^{2}} q^{\mu}
$$

It would then be possible to achieve a gauge invariant result, while keeping $m_{q}^{\prime} \neq m_{q}$. 
2. By analogy with the treatment of $\pi^{0}$-Photoproduction on a quark, one can restore gauge invariance by including the crossed Feynman diagram shown in Fig. 3, in which, in the pion-quark coupling $g_{\pi} \gamma_{5}$, the constant $g_{\pi}$ is replaced by a phenomenological scalar function. This was tried by CL [8], but they were unable to reproduce the standard result in the limit $Q^{2} \rightarrow \infty$.

\section{CONCLUSIONS}

Guerrero, Ethier, Accardi, Casper and Melnitchouk have produced a study of semiinclusive deep inelastic scattering, which attempts to take into account the masses of the target and produced hadron, contrary to the conventional treatment which ignores all hadronic

masses. They then argue that such effects might reduce the apparent discrepancy between the HERMES and COMPASS pion and kaon multiplicity measurements.

Unfortunately it turns out that the GEACM analysis is inconsistent, in that terms of the same order of magnitude as those they are concerned about, are neglected. Moreover, when such terms are included the resulting hadronic tensor is no longer gauge invariant.

We have, regrettably, been forced to conclude, that in contradistinction to inclusive DIS, where it is possible to calculate exact target mass corrections, attempts to include kinematic hadron mass corrections in semi-inclusive DIS run into insurmountable difficulties. It seems that the standard formulation of a semi-inclusive event, as a product of a parton density times an independent fragmentation function does not work if hadron masses are taken into account.

\section{ACKNOWLEDGMENTS}

E.L. is grateful to the Leverhulme Trust for an Emeritus Fellowship.

[1] O. Nachtmann, Nucl. Phys., B63, 237 (1973).

[2] H. Georgi and H. D. Politzer, Phys. Rev., D14, 1829 (1976).

[3] S. Matsuda and T. Uematsu, Nucl. Phys., B168, 181 (1980).

[4] S. Wandzura, Nucl. Phys., B122, 412 (1977). 
[5] A. Piccione and G. Ridolfi, Nucl. Phys., B513, 301 (1998), hep-ph/9707478.

[6] J. Blumlein and A. Tkabladze, Nucl. Phys., B553, 427 (1999), hep-ph/9812478.

[7] U. DAlesio, E. Leader, and F. Murgia, Phys. Rev., D81, 036010 (2010), arXiv:0909.5650 [hep-ph].

[8] K. Christova and E. Leader, Hadron mass corrections to sidis, unpublished, (2011).

[9] J. V. Guerrero, J. J. Ethier, A. Accardi, S. W. Casper, and W. Melnitchouk, JHEP, 09, 169 (2015), arXiv:1505.02739 [hep-ph].

[10] A. Accardi, "See Extra Slides in Talk presented at QCDN'16, Getxo, Spain" (July, 2016)

[11] J. Levelt and P. J. Mulders, Phys. Rev., D49, 96 (1994), arXiv:hep-ph/9304232 [hep-ph].

[12] P. J. Mulders and R. D. Tangerman, Nucl. Phys., B461, 197 (1996).

[13] R. K. Ellis, W. Furmanski, and R. Petronzio, Nucl. Phys., B212, 29 (1983). 